https://helda.helsinki.fi

Physical activity and anxiety : A perspective from the World Health Survey

\title{
Stubbs, Brendon
}

2017-01-15

Stubbs , B , Koyanagi , A , Hallgren, M , Firth , J , Richards , J , Schuch , F , Rosenbaum , S , Mugisha, J , Veronese , N , Lahti , J \& Vancampfort, D 2017 , ' Physical activity and anxiety : A perspective from the World Health Survey ', Journal of Affective Disorders, vol. 208 , pp. 545-552 . https://doi.org/10.1016/j.jad.2016.10.028

http://hdl.handle.net/10138/231347

https://doi.org/10.1016/j.jad.2016.10.028

publishedVersion

Downloaded from Helda, University of Helsinki institutional repository.

This is an electronic reprint of the original article.

This reprint may differ from the original in pagination and typographic detail.

Please cite the original version. 


\title{
Physical activity and anxiety: A perspective from the World Health Survey
}

\author{
Brendon Stubbs ${ }^{\mathrm{a}, \mathrm{b}, *}$, Ai Koyanagi ${ }^{\mathrm{c}, \mathrm{d}}$, Mats Hallgren ${ }^{\mathrm{e}}$, Joseph Firth ${ }^{\mathrm{f}}$, Justin Richards ${ }^{\mathrm{g}}$, \\ Felipe Schuch ${ }^{\mathrm{h}, \mathrm{i}}$, Simon Rosenbaum ${ }^{\mathrm{j}}$, James Mugisha ${ }^{\mathrm{k}, \mathrm{l}}$, Nicola Veronese ${ }^{\mathrm{m}, \mathrm{n}}$, Jouni Lahti ${ }^{\mathrm{o}}$, \\ Davy Vancampfort ${ }^{\mathrm{p}, \mathrm{q}}$
}

a Physiotherapy Department, South London and Maudsley NHS Foundation Trust, Denmark Hill, London SE5 8AZ, United Kingdom

${ }^{\mathrm{b}}$ Health Service and Population Research Department, Institute of Psychiatry, Psychology and Neuroscience, King's College London, De Crespigny Park, London Box SE5 8AF, United Kingdom

${ }^{\mathrm{c}}$ Research and Development Unit, Parc Sanitari Sant Joan de Déu, Universitat de Barcelona, Fundació Sant Joan de Déu, Dr. Antoni Pujadas, 42 , Sant Boi de Llobregat, Barcelona 08830, Spain

' Instituto de Salud Carlos III, Centro de Investigación Biomédica en Red de Salud Mental, CIBERSAM, Monforte de Lemos 3-5 Pabellón 11, Madrid 28029, Spain

e Division of Epidemiology and Public Health Intervention Research (EPHIR), Department of Public Health Sciences, Karolinska Institutet, Solna 171 77,

Sweden

${ }^{\mathrm{f}}$ Institute of Brain, Behaviour and Mental Health, University of Manchester, Manchester, United Kingdom

g School of Public Health and Charles Perkins Centre, University of Sydney, Sydney, NSW, Australia

${ }^{\mathrm{h}}$ Hospital de Clínicas de Porto Alegre, Porto Alegre, Brazil

i Pós Graduação em Ciências Médicas Unilassale, Canoas, Brazil

${ }^{\mathrm{j}}$ Department of Exercise Physiology, School of Medical Sciences, University of New South Wales, Sydney, Australia

${ }^{\mathrm{k}}$ Kyambogo University, Kampala, Uganda

${ }^{1}$ Butabika National Referral and Mental Health Hospital, Kampala, Uganda

$\mathrm{m}$ Geriatrics Division, Department of Medicine-DIMED, University of Padova, Italy

${ }^{n}$ Institute of clinical Research and Education in Medicine (IREM), Padova, Italy

o Department of Public Health, University of Helsinki, Helsinki, Finland

p KU Leuven Department of Rehabilitation Sciences, Leuven, Belgium

${ }^{\mathrm{q}}$ KU Leuven, University Psychiatric Center KU Leuven, Leuven-Kortenberg, Belgium

\section{A R T I C L E I N F O}

\section{Keywords:}

Physical activity

Anxiety

Exercise

Multi-country study

Community-based

Psychiatry

\begin{abstract}
A B S T R A C T
Background: Despite the known benefits of physical activity (PA) among people with anxiety, little is known about PA levels in people with anxiety at the population level. This study explored the global prevalence of anxiety and its association with PA.

Methods: Cross-sectional, community-based data from the World Health Survey was analyzed. Prevalence of anxiety was estimated for 237,964 individuals (47 countries). PA was categorized as low, moderate, and high based on the International Physical Activity Questionnaire (short form). The association between PA and anxiety was assessed by multivariable logistic regression.

Results: The overall global prevalence of anxiety was $11.4 \%$ (47 countries). Across 38 countries with available data on PA, $62.5 \%, 20.2 \%$, and $17.3 \%$ of the sample engaged in high, moderate, and low levels of PA respectively. The prevalence of low physical activity in those with and without anxiety was $22.9 \%$ vs. $16.6 \%$ ( $p<$ 0.001) (38 countries, $n=184,920$ ). In the pooled model adjusted for socio-demographics, depression, and country, individuals engaging in low PA (vs. high PA) had 1.32 (95\% CI=1.17-1.47) times higher odds for anxiety than those with high PA. Female sex, older age, lower education and wealth, and depression were also associated with low PA. At the individual country level, there was a significant positive association between low PA and anxiety in 17 of the 38 countries.

Conclusion: Low PA levels are associated with increased prevalence of anxiety. There is a need for longitudinal research to establish the directionality of the relationships observed.
\end{abstract}

\footnotetext{
* Corresponding author at: Physiotherapy Department, South London and Maudsley NHS Foundation Trust, Denmark Hill, London, United Kingdom.

E-mail address: brendon.stubbs@kcl.ac.uk (B. Stubbs).
} 


\section{Introduction}

Anxiety is a common and burdensome mental health complaint (Haller et al., 2014) which can greatly impair an individual's functioning, quality of life and well-being (Mendlowicz and Stein, 2000; Olatunji et al., 2007). Symptoms of anxiety are personified by nervousness, pervasive thoughts of worry and pessimism, which if left untreated can develop into an anxiety disorder (Herring et al., 2010). The global prevalence of anxiety disorders ranges from $2.4-29.8 \%$ in the past year (Baxter et al., 2013), whilst high prevalence of subthreshold anxiety (Haller et al., 2014) and symptoms of anxiety are also common and problematic across the lifespan (Prina et al., 2011; Stubbs et al., 2016a; Baxter et al., 2014). Anxiety disorders are the sixth leading cause of disability, in terms of years lived with disability, in both high-income and low- and middle-income countries. Globally, anxiety disorders accounted for 390 disability-adjusted life years per 100,000 persons in 2010 , with no discernible change observed over time (Baxter et al., 2014). The burden of anxiety is increased even more by the high risk of co-morbid cardiovascular diseases and associated premature mortality (Batelaan et al., 2016; Roest et al., 2012). Therefore, it is not a surprise that the global financial burden of anxiety is substantial, owing to lost work productivity and high medical resource use (Chisholm et al., 2016; Hoffman et al., 2008).

Antidepressants such as selective serotonin reuptake inhibitor (SSRI) or serotonin-norepinephrine reuptake inhibitor (SNRI) or benzodiazepines (Baldwin et al., 2011, 2010) are the frontline treatment of anxiety symptoms among people with anxiety disorders (de Vries et al., 2016) while cognitive behavioral therapy is efficacious in reducing anxiety symptoms (Hofmann and Smits, 2008). More recently, evidence has been accumulating that self-help interventions may also be beneficial in people with anxiety disorders (Lewis et al., 2012). For example, physical activity has been proposed as such a selfmanagement strategy (Pedersen and Saltin, 2015; Jayakody et al., 2013; Conn, 2010). Previous systematic reviews (Pedersen and Saltin, 2015; Jayakody et al., 2013; Conn, 2010) have suggested that physical activity is helpful in reducing anxiety symptoms, while its role in preventing cardio-metabolic risks is largely known (Stubbs et al., Rosenbaum). People with anxiety may be at increased risk of cardiovascular disease and poorer cardiorespiratory function (Caldirola et al., 2016) and physical activity may help improve these outcomes (Vancampfort et al., 2016a). Despite the established benefits of physical activity in people with anxiety, little is known about physical activity levels in this population. Studies that have considered the relationship between physical activity and anxiety have been of small sample sizes and limited to one country (Helgadóttir et al., 2015; da Silva et al., 2014; Da Silva et al., 2012; Park et al., 2011). In addition, at the population level, there is a paucity of information on factors that might influence physical activity and anxiety. Such information might prove useful for developing interventions.

Given the aforementioned, using multi-country data from the World Health Survey, the current paper set out with the following aims: (a) describe the concurrent global prevalence of physical activity and anxiety; and (b) explore the association between anxiety and physical activity.

\section{Methods}

\subsection{The survey}

Data from the current study derives from the World Health Survey (WHS). The WHS was a cross-sectional, community-based survey carried out in 70 countries between 2002 and 2004. Data were collected using single-stage random sampling in 10 countries, while stratified multi-stage random cluster sampling was employed in 60 countries. Survey details are available elsewhere (http://www.who.int/ healthinfo/survey/en/). Briefly, individuals with a valid home address aged $\geq 18$ years were eligible to participate. The questionnaire was subject to standard translation procedures to ensure comparability across languages and cultures. Information was obtained through faceto-face and telephone interviews conducted by trained interviewers. Across all countries, the individual response rate was $98.5 \%$ (Nuevo et al., 2012). To adjust for non-response, sampling weights were generated using the population distribution as reported by the United Nations Statistical Division. Ethical approval for the survey was provided by ethical boards at each study site. All participants provided their informed consent.

\subsection{Variables}

\subsubsection{Anxiety (outcome variable)}

Anxiety was assessed by the question 'Overall in the past 30 days, how much of a problem did you have with worry or anxiety' with answer options being none, mild, moderate, severe, and extreme. In accordance with previous WHS publications, those who answered severe and extreme were considered to have anxiety within the current study (Koyanagi and Stickley, 2015; Wong et al., 2013).

\subsubsection{Physical activity (exposure variable)}

Physical activity was assessed using the short form of the International Physical Activity Questionnaire (C.L. Craig et al., 2003; C. Craig et al., 2003), in which respondents are asked to report the number of days and the duration of the vigorous, moderate, and walking activities they undertook during the last 7 days. Show-cards illustrating different types of vigorous and moderate physical activities were presented to the respondents in addition to brief explanations of what was meant by vigorous and moderate activity. The summary indicator of the IPAQ was used to categorize the overall population into three levels of physical activity: low, moderate or high. The moderate level nominally indicated meeting any of the following three criteria: (a) 3 days of vigorous activity of at least $20 \mathrm{~min} /$ day; (b) 5 days of moderate-intensity activity or walking of $>30 \mathrm{~min} /$ day for $>10 \mathrm{~min}$ at a time; or (c) 5 days of any combination of walking, moderate-intensity or vigorous-intensity activities achieving at least 600 MET-minutes/ week. High levels of physical activity are defined as meeting either of two criteria: (a) vigorous-intensity activity on $>3$ days/week and accumulating at least 1500 MET-minutes/week; or (b) $>5$ days of any combination of walking, moderate-intensity, or vigorous-intensity activities achieving at least 3000 MET-minutes/week.

One MET is defined as $1 \mathrm{kcal} / \mathrm{kg} / \mathrm{h}$ and is more or less equivalent to the energy cost of sitting quietly (C.L. Craig et al., 2003; C. Craig et al., 2003). Those who neither meet the moderate nor high criteria were categorized in the low level physical activity group. These category definitions correspond to the criteria used for classifying people as insufficiently active in the largest study of global physical activity levels to date (Hallal et al., 2012).

\subsubsection{Control variables}

Information on sex, age (18-34, 35-59, $\geq 60$ years), education, wealth, and depression were used for adjustment. Education was categorized as: no formal education, primary education, secondary or high school completed, or tertiary education completed. Principal component analysis based on 15-20 assets was conducted to create country-wise wealth quintiles. The DSM-IV algorithm was used to identify depression based on the duration and persistence of past-12 month depressive symptoms (Koyanagi and Stickley, 2015).

\subsubsection{Statistical analysis}

The statistical analysis was done with Stata 14.1 (Stata Corp LP, College station, Texas). Of the 69 countries for which data were publically available, 10 countries with no sampling information (Austria, Belgium, Denmark, Germany, Greece, Guatemala, Italy, Netherlands, Slovenia, UK) were excluded. We subsequently excluded 
12 countries (Congo, France, Finland, Ireland, Israel, Luxembourg, Morocco, Norway, Portugal, Slovakia, Swaziland, Sweden) as they either did not have information on anxiety, or because $>25 \%$ of the data on anxiety was missing. We calculated the age- and sex-adjusted prevalence of anxiety for all 237,964 individuals aged $\geq 18$ years in these 47 countries. For all other analyses, we further excluded 9 countries (Chad, Ecuador, Ethiopia, Latvia, Mali, Mauritania, Senegal, Turkey, Ukraine), which were either completely missing data on physical activity or had $>25 \%$ of the data missing. Thus, in all subsequent analyses, 38 countries were included, and we restricted the sample to those aged 18-69 years since the IPAQ is considered to be valid for this age range (C. Craig et al., 2003; C.L. Craig et al., 2003). The sample size after this restriction was 184,920. Age- and sexadjusted estimates for anxiety and physical activity for each country were estimated by using the United Nations population pyramids for the year 2010 (http://esa.un.org/wpp/Excel-Data/population.htm) as the standard population. The difference in sample characteristics between those with and without anxiety was tested by Chi-squared tests. Using the pooled sample, multivariable logistic regression analysis was done to assess the association between physical activity (low, moderate, high) and anxiety. We constructed 4 models to assess the influence of the inclusion of different covariates into the models: Model 1 - adjusted for country; Model 2 - adjusted for sex, age, and country; Model 3 - adjusted for sex, age, education, wealth, and country; Model 4 - adjusted for sex, age, education, wealth, depression, and country. Adjustment for country was done by including dummy variables in the models, as in previous WHS publications (Nuevo et al., 2012; Koyanagi and Stickley, 2015). We also tested for effect modification by gender and age groups (18-34, $35-59, \geq 60$ years) in the association between low PA and anxiety by including interaction terms (i.e. low PA X gender, or low PA X age group) in the fully adjusted model (Model 4).

Finally, country-wise multivariable logistic regression models were constructed to assess the association between low physical activity and anxiety. The estimates for each country were also combined into a random-effect meta-analysis. This was done to assess the generalizability of our findings across countries. Based on the results of the pooled analysis, we decided to: (1) collapse the high and moderate levels of physical activity for country-wise analyses as the association between anxiety were similar for these two categories; and (2) adjust only for age and sex as other variables had little influence in the association between physical activity and anxiety. The sample weighting and the complex study design were taken into account in all analyses. Results from the logistic regression models are presented as odds ratios (ORs) with 95\% confidence intervals (CIs). The level of statistical significance was set at $P<0.05$.

\section{Results}

The country sample size ranged from 929 (Latvia) to 38,746 (Mexico) (Table 1). The overall crude prevalence of anxiety in 47 countries was $11.4 \%$ (95\%CI $11.1-11.8 \%$ ). The age- and sex-adjusted prevalence of anxiety varied widely between countries and ranged from 1.1\% (China, Vietnam) to 79.2\% (Kazakhstan), with Sri Lanka (64.9\%), Brazil (22.4\%), and Bangladesh (20.9\%) also having high rates of anxiety. The country-wise prevalence of anxiety is graphically displayed in Fig. 1. Most countries in Asia had low prevalence of anxiety with the exception of the Indian subcontinent, Kazakhstan, and Turkey. Africa had a mix of low and high rates of anxiety, while higher prevalence were observed in South-America.

In terms of physical activity, of the 38 countries, the highest ageand sex-adjusted prevalence of low physical activity were observed in United Arab Emirates (50.9\%), South Africa (47.6\%), Dominican Republic (42.4\%), and Namibia (40.5\%). The characteristics of the sample restricting to 18-69 years (38 countries) are shown in Table 2. There were slightly more females than males $(50.2 \%$ vs. $49.8 \%)$ with
Table 1

Age- and sex-adjusted prevalence of physical activity and anxiety by country.

\begin{tabular}{|c|c|c|c|c|c|}
\hline \multirow[b]{2}{*}{ Country } & \multirow[b]{2}{*}{$N$} & \multicolumn{3}{|c|}{ Physical activity } & \multirow[t]{2}{*}{ Anxiety } \\
\hline & & High & Moderate & Low & \\
\hline Bangladesh & 5942 & $63.6(1.4)$ & $18.5(0.9)$ & $18.0(1.0)$ & $20.9(1.0)$ \\
\hline Bosnia Herzegovina & 1031 & $62.8(4.1)$ & $21.7(2.5)$ & $15.5(4.3)$ & $11.3(1.5)$ \\
\hline Brazil & 5000 & $47.2(1.1)$ & $24.4(0.8)$ & $28.4(1.0)$ & $22.4(0.7)$ \\
\hline Burkina Faso & 4948 & $73.6(1.6)$ & $17.4(1.2)$ & $9.1(0.9)$ & $9.5(1.1)$ \\
\hline Chad & 4870 & & & & $6.2(0.6)$ \\
\hline China & 3994 & $62.0(4.2)$ & $27.4(2.5)$ & $10.6(1.8)$ & $1.1(0.2)$ \\
\hline Comoros & 1836 & $87.6(1.6)$ & $9.3(1.3)$ & $3.0(0.7)$ & $15.4(1.2)$ \\
\hline Croatia & 993 & 70.7 (1.9) & $20.8(1.7)$ & $8.5(1.1)$ & $8.9(1.2)$ \\
\hline Czech Republic & 949 & $68.5(3.1)$ & $22.2(3.0)$ & $9.3(1.6)$ & $6.9(1.4)$ \\
\hline Dominican Republic & 5027 & $31.2(1.3)$ & $26.4(1.1)$ & $42.4(1.3)$ & $10.7(0.9)$ \\
\hline Ecuador & 5675 & & & & $9.1(0.8)$ \\
\hline Estonia & 1020 & $83.1(2.1)$ & $12.1(1.2)$ & $4.8(1.1)$ & $6.3(1.0)$ \\
\hline Ethiopia & 5089 & & & & $11.6(0.7)$ \\
\hline Georgia & 2950 & $69.0(2.7)$ & $22.4(2.3)$ & $8.6(1.2)$ & $6.0(0.8)$ \\
\hline Ghana & 4165 & $65.0(1.3)$ & $21.9(1.0)$ & $13.1(1.0)$ & $6.4(0.5)$ \\
\hline Hungary & 1419 & $76.5(1.7)$ & $15.1(1.2)$ & $8.4(1.1)$ & $6.8(0.7)$ \\
\hline India & 10687 & 73.9 (1.6) & $11.8(0.7)$ & $14.3(1.7)$ & $13.2(1.0)$ \\
\hline Ivory Coast & 3251 & $62.0(2.0)$ & $22.8(1.6)$ & $15.2(1.4)$ & $8.4(0.8)$ \\
\hline Kazakhstan & 4499 & $58.9(3.7)$ & $27.9(2.4)$ & $13.2(2.0)$ & $79.2(1.3)$ \\
\hline Kenya & 4640 & $78.6(1.9)$ & $11.0(1.0)$ & $10.4(1.4)$ & $6.3(0.7)$ \\
\hline Laos & 4988 & $68.7(1.0)$ & $18.3(0.8)$ & $13.0(0.8)$ & $2.6(0.3)$ \\
\hline Latvia & 929 & & & & $11.7(1.4)$ \\
\hline Malawi & 5551 & $69.9(1.6)$ & $18.7(1.1)$ & $11.4(1.0)$ & $13.5(0.7)$ \\
\hline Malaysia & 6145 & $51.6(1.0)$ & $28.2(0.8)$ & $20.2(0.7)$ & $1.8(0.3)$ \\
\hline Mali & 4886 & & & & $6.2(0.5)$ \\
\hline Mauritania & 3902 & & & & $3.7(0.5)$ \\
\hline Mauritius & 3968 & $52.8(2.2)$ & $28.9(1.4)$ & $18.3(1.5)$ & $11.0(0.8)$ \\
\hline Mexico & 38746 & $61.6(0.7)$ & $20.6(0.4)$ & $17.7(0.5)$ & $5.2(0.2)$ \\
\hline Myanmar & 6045 & $60.7(2.1)$ & $27.9(1.6)$ & $11.3(1.3)$ & $2.2(0.4)$ \\
\hline Namibia & 4379 & 38.5 (1.4) & $20.9(1.0)$ & $40.5(1.5)$ & $9.5(0.8)$ \\
\hline Nepal & 8820 & $77.1(0.7)$ & $13.5(0.6)$ & $9.5(0.4)$ & $17.1(0.6)$ \\
\hline Pakistan & 6501 & $50.9(1.1)$ & $27.4(1.3)$ & $21.7(1.0)$ & $3.8(0.3)$ \\
\hline Paraguay & 5288 & $50.6(1.0)$ & $28.9(0.9)$ & $20.5(0.9)$ & $12.7(0.6)$ \\
\hline Philippines & 10083 & $75.8(1.0)$ & $16.8(0.8)$ & $7.4(0.6)$ & $4.4(0.4)$ \\
\hline Russia & 4427 & $77.0(2.2)$ & $17.2(2.0)$ & $5.8(0.9)$ & $6.9(0.8)$ \\
\hline Senegal & 3461 & & & & $6.1(0.6)$ \\
\hline South Africa & 2629 & $29.8(2.1)$ & $22.6(1.4)$ & $47.6(2.3)$ & $13.6(1.1)$ \\
\hline Spain & 6373 & $34.3(1.2)$ & $35.4(1.0)$ & $30.3(1.0)$ & $7.2(0.5)$ \\
\hline Sri Lanka & 6805 & 70.2 (1.6) & $19.3(1.0)$ & $10.6(1.1)$ & $64.9(1.8)$ \\
\hline Tunisia & 5202 & $58.6(1.7)$ & $25.6(1.2)$ & $15.8(1.1)$ & $9.3(0.7)$ \\
\hline Turkey & 11481 & & & & $13.9(0.5)$ \\
\hline Ukraine & 2860 & & & & $7.2(0.8)$ \\
\hline United Arab Emirates & 1183 & $14.0(1.7)$ & $35.1(2.8)$ & $50.9(3.0)$ & $4.6(1.1)$ \\
\hline Uruguay & 2996 & $39.2(4.2)$ & $33.9(3.1)$ & $26.9(2.4)$ & $10.3(1.2)$ \\
\hline Vietnam & 4174 & $78.2(2.2)$ & $13.1(1.5)$ & $8.7(1.4)$ & $1.1(0.2)$ \\
\hline Zambia & 4165 & $63.1(1.5)$ & $25.3(1.2)$ & $11.6(0.9)$ & $7.1(0.6)$ \\
\hline Zimbabwe & 4290 & $64.5(1.3)$ & $15.9(0.9)$ & $19.7(1.0)$ & $11.3(0.9)$ \\
\hline
\end{tabular}

Data are unweighted $\mathrm{N}$ and weighted \% (SE).

All age- and sex-adjusted estimates were calculated using the United Nations population pyramids for the year 2010 .

a Estimates for physical activity is restricted to those aged 18-69 years.

approximately half the sample being of age 18-34 years. Overall, $62.5 \%, 20.2 \%$, and $17.3 \%$ engaged in high, moderate, and low levels of physical activity respectively. The prevalence of low physical activity in those with and without anxiety was $22.9 \%$ vs. $16.6 \%(p<0.001)$. Female sex, older age, low socio-economic status (education and wealth), and depression were also associated with higher prevalence of anxiety (Table 2).

\subsection{Association between physical activity and anxiety}

The association between physical activity and anxiety estimated by logistic regression is shown in Table 3. In the model only adjusted for country, when compared to those with high levels of physical activity, individuals engaging in low physical activity had 1.47 (95\%CI $=1.33-$ 1.62) times higher odds of anxiety (Model 1), while there was no significant difference for those with moderate levels of physical activity. 


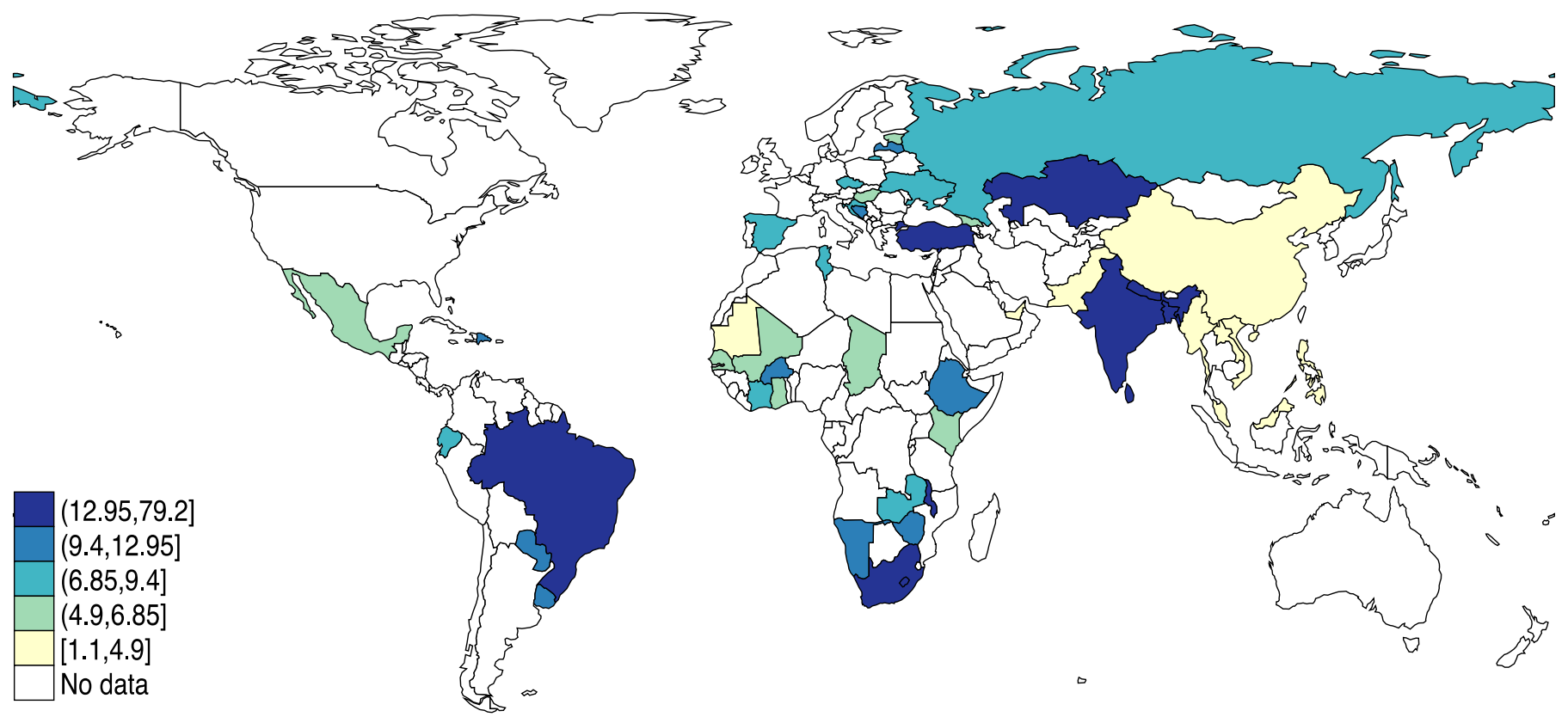

Fig. 1. Age- and sex-adjusted prevalence (\%) of anxiety in 47 countries.Estimates were calculated using the United Nations population pyramids for the year 2010.

Table 2

Sample characteristics of individuals aged 18-69 years in 38 countries.

\begin{tabular}{lllll}
\hline \multirow{2}{*}{ Characteristic } & Category & Total & $\begin{array}{l}\text { Anxiety } \\
\text { No }\end{array}$ & Yes \\
\hline \multirow{2}{*}{ Physical activity } & High & 62.5 & 63.1 & 58.0 \\
& Moderate & 20.2 & 20.3 & 19.1 \\
& Low & 17.3 & 16.6 & 22.9 \\
& Male & & & \\
Sex & Female & 49.8 & 51.0 & 40.2 \\
& & 50.2 & 49.0 & 59.8 \\
Age (years) & 18-34 & 50.3 & 51.7 & 39.0 \\
& 35-59 & 41.8 & 40.8 & 49.8 \\
& $\geq 60$ & 8.0 & 7.6 & 11.2 \\
& & & & \\
Education & No formal & 23.0 & 22.8 & 25.0 \\
& $\leq$ Primary & 32.1 & 31.8 & 34.5 \\
& Secondary completed & 35.4 & 35.8 & 32.2 \\
& Tertiary completed & 9.4 & 9.6 & 8.4 \\
Wealth & & & & \\
& Poorest & 19.4 & 19.0 & 22.7 \\
& Poorer & 19.8 & 19.6 & 21.3 \\
& Middle & 20.1 & 20.1 & 19.6 \\
& Richer & 20.3 & 20.5 & 19.2 \\
& Richest & 20.4 & 20.8 & 17.1 \\
& & 6.6 & 4.4 & 24.5 \\
\hline \multirow{2}{*}{ Yes } & & &
\end{tabular}

Data are column \%. Estimates are based on weighted sample.

$P<0.0001$ (Chi-squared test) for all differences in sample characteristics between those with and without anxiety.

The OR of low physical activity was slightly attenuated when adjusted for age and sex (Model 2) but further adjustment for wealth, education, and depression had little influence on the association (Model 3 and 4). In the fully-adjusted model, the OR (95\%CI) was 1.32 (95\%CI=1.171.47) (Model 4). There were no significant interactions for the association between low PA and anxiety for sex or age groups. The country-wise association between low physical activity and anxiety is illustrated in Fig. 2. The OR (95\%CI) ranged from $0.13(0.02-0.93)$ in Vietnam to $3.36(1.40-8.07)$ in China with the pooled estimate being 1.43 (1.27-1.62). There was a significant positive association between low physical activity and anxiety in 17 of the 38 countries.

\section{Discussion}

Our paper reiterates the high prevalence and disease burden of anxiety globally. To the best of our knowledge, our analyses are the first to suggest on a global level that engaging in low physical activity levels (i.e. being physically inactive) is associated with anxiety. Specifically, after full-adjustment, compared to high physical activity, individuals engaging in low physical activity had $32 \%$ higher odds of experiencing anxiety, while there was no significant difference for moderate levels of physical activity. Low levels of physical activity, female sex, older age, lower socio-economic status (education and wealth), and depression were associated with a higher prevalence of anxiety. In addition, our country-wide meta-analysis exploring the association between low physical activity and anxiety demonstrated a pooled adjusted OR of $1.43(95 \% \mathrm{CI}=1.27-1.62)$ with a significantly positive association being observed in 17 of the 38 countries.

In our study, age, gender, wealth, education, and depression did not have a large influence on the association between PA and anxiety. This suggests that other factors might play a role. Previous research in Taiwan indicated that in young children with anxiety, physical activity participation is significantly correlated with perceived physical benefits, higher levels of self-efficacy, more social support, and less reported barriers (Ma et al., 2009). These are consistent with evidence for healthy individuals and may be important factors that were not measured in our study but are key factors to increase physical activity among those with anxiety. Also, previous research in people with panic disorder identified that higher levels of somatic, but not cognitive symptoms may prevent physical activity behavior (da Silva et al., 2014). Clearly, representative research investigating physical activity levels and anxiety is in its infancy and future longitudinal research should explore the directionality and factors influencing this relationship. In particular, next to exploring the role of self-efficacy and social support on physical activity levels in people with anxiety on a global level, culture-specific benefits of and barriers for physical activity should be investigated in this population. Understanding such factors may result in the development of population level and community specific interventions to increase physical activity that are inclusive of people experiencing anxiety. Considering the benefits of physical activity on wellbeing (Penedo and Dahn, 2005), cardiovascular disease and mortality (Penedo and Dahn, 2005), it is important that population-level interventions are developed in order to increase physical 
Table 3

The association between physical activity and anxiety estimated by multivariable logistic regression.

\begin{tabular}{|c|c|c|c|c|c|c|c|c|c|}
\hline \multirow[b]{2}{*}{ Characteristic } & \multicolumn{3}{|c|}{ Model 1} & \multicolumn{2}{|c|}{ Model 2} & \multicolumn{2}{|c|}{ Model 3} & \multicolumn{2}{|c|}{ Model 4} \\
\hline & OR & $95 \% \mathrm{CI}$ & & OR & $95 \% \mathrm{CI}$ & OR & $95 \% \mathrm{CI}$ & OR & $95 \% \mathrm{CI}$ \\
\hline \multicolumn{10}{|l|}{ Physical activity } \\
\hline High & 1.00 & & {$[1.00,1.00]$} & 1.00 & {$[1.00,1.00]$} & 1.00 & {$[1.00,1.00]$} & 1.00 & {$[1.00,1.00]$} \\
\hline Moderate & 1.01 & & {$[0.92,1.11]$} & 0.92 & {$[0.84,1.01]$} & 0.98 & {$[0.89,1.07]$} & 0.99 & {$[0.89,1.09]$} \\
\hline Low & $1.47^{* * * *}$ & & {$[1.33,1.62]$} & $1.30^{* * * * *}$ & {$[1.18,1.43]$} & $1.34^{* * * *}$ & {$[1.21,1.48]$} & $1.32^{* * * *}$ & {$[1.17,1.47]$} \\
\hline \multicolumn{10}{|l|}{ Sex } \\
\hline Male & & & & 1.00 & {$[1.00,1.00]$} & 1.00 & {$[1.00,1.00]$} & 1.00 & {$[1.00,1.00]$} \\
\hline Female & & & & $1.69^{* * * * *}$ & {$[1.57,1.83]$} & $1.68^{* * *}$ & {$[1.55,1.82]$} & $1.56^{* * * *}$ & {$[1.44,1.70]$} \\
\hline \multicolumn{10}{|l|}{ Age (years) } \\
\hline $18-34$ & & & & 1.00 & {$[1.00,1.00]$} & 1.00 & {$[1.00,1.00]$} & 1.00 & {$[1.00,1.00]$} \\
\hline $35-59$ & & & & $1.63^{* * * *}$ & {$[1.51,1.75]$} & $1.58^{* * * *}$ & {$[1.46,1.71]$} & $1.46^{* * * *}$ & {$[1.33,1.59]$} \\
\hline$\geq 60$ & & & & $2.02^{* * * *}$ & {$[1.80,2.26]$} & $1.86^{* * * *}$ & {$[1.65,2.09]$} & $1.63^{* \ldots * *}$ & {$[1.44,1.86]$} \\
\hline \multicolumn{10}{|l|}{ Education } \\
\hline No formal & & & & & & 1.00 & {$[1.00,1.00]$} & 1.00 & {$[1.00,1.00]$} \\
\hline$\leq$ Primary & & & & & & 0.96 & {$[0.85,1.07]$} & 0.99 & {$[0.88,1.13]$} \\
\hline Secondary completed & & & & & & $0.78^{* * * *}$ & {$[0.68,0.89]$} & $0.81^{* * *}$ & {$[0.70,0.94]$} \\
\hline Tertiary completed & & & & & & $0.69^{* * * *}$ & {$[0.57,0.84]$} & $0.79^{*}$ & {$[0.63,0.98]$} \\
\hline \multicolumn{10}{|l|}{ Wealth } \\
\hline Poorest & & & & & & 1.00 & {$[1.00,1.00]$} & 1.00 & {$[1.00,1.00]$} \\
\hline Poorer & & & & & & 0.94 & {$[0.86,1.04]$} & 0.94 & {$[0.85,1.05]$} \\
\hline Middle & & & & & & $0.85^{* * *}$ & {$[0.76,0.94]$} & $0.84^{* * *}$ & {$[0.75,0.94]$} \\
\hline Richer & & & & & & $0.85^{* *}$ & {$[0.76,0.95]$} & $0.86^{*}$ & {$[0.76,0.97]$} \\
\hline Richest & & & & & & $0.75^{* * * *}$ & {$[0.66,0.86]$} & $0.79^{* * * *}$ & {$[0.69,0.91]$} \\
\hline Depression & & & & & & & & $6.11^{* \ldots * *}$ & {$[5.45,6.85]$} \\
\hline
\end{tabular}

Abbreviation: OR odds ratio; CI confidence interval

Estimates are based on data from individuals aged 18-69 years from 38 countries.

Models are adjusted for all variables in the respective column and country.

${ }^{*} p<0.05$

${ }^{* * *} p<0.01$

**** $p<0.001$

activity and take into account the high prevalence of anxiety globally.

The fact that no age- or gender-interaction effects were found points to the need to prescribe physical activity across the lifespan, at the earliest stages, and for both genders. At the other end of the physical activity spectrum, recent systematic reviews have suggested that higher levels of sedentary behavior are associated with an increased risk of future anxiety in people without depression (Teychenne et al., 2010, 2015). Moreover, recent literature in adolescents has attempted to disentangle the potential relationships, and found that experimentally-induced sedentary behavior among previously active young adults is associated with increased anxiety levels (Edwards and Loprinzi, 2016). Therefore, sedentary behavior, even at a young age, may lead to anxiety through biological pathways (Edwards and Loprinzi, 2016). For example, engaging in screen-based sedentary behaviors, has been shown to increase the arousal of the central nervous system in children (Wang and Perry, 2006), which could in turn increase anxiety (Wang and Perry, 2006). Additionally, screenbased entertainment has also been linked to disrupted sleeping patterns, which may also elevate levels of anxiety (Dworak et al., 2007). An earlier randomized control trial of experimentally induced sedentary behavior demonstrated that increases in sedentary behavior were associated with lower mood, which was explained from a worsening inflammatory profile (Endrighi et al., 2015). Although we recognize that sedentary behavior has been identified as a separate risk factor to insufficient physical activity for several health-related outcomes, it is widely accepted that these two behaviors often occur in tandem across the lifespan and are likely to have similar mental health implications (Teychenne and York, 2013; Rebar et al., 2014; Hoare et al., 2014).

Another potential hypothesis of the link between low physical activity and anxiety may be explained by the social withdrawal theory.
This posits that not engaging in physical activities may lead to social solitude and withdrawal from interpersonal relationships, both of which have been linked to increased feelings of social anxiety (Rubin, 2001). On the other hand, it may be that those with anxiety are less inclined to engage in physical activity as a means of coping with their anxiety symptoms, as has been suggested in previous research amongst people with mental illness with social physique anxiety (De Herdt et al., 2013). However, more research on the role of social anxiety on physical activity participation across different cultural settings is now needed to make any firm conclusions. Large scale representative data should explore the prospective relationship between physical activity and anxiety.

\subsection{Practical implications}

The current data highlight that anxiety is a common problems across the world and is associated with low levels of physical activity. Policy makers should invest in promoting physical activity at a population level as well as through adapted services that are most likely to reach people experiencing anxiety. This may include the provision of specific training to improve the mental health awareness and intervention delivery skills of clinicians most commonly involved with physical activity promotion, such as medical personnel, physiotherapists and exercise physiologists (Stubbs et al., 2016b; Vancampfort et al., 2016b). These providers should be able to recognize the signs and symptoms of anxiety and demonstrate basic knowledge of the causes. It is also important that the current workforce understand the most effective methods for implementing a behavioral intervention in this sub-population, which requires further investigation in relation to physical activity promotion.

Research should explore the most optimal form of physical activity 


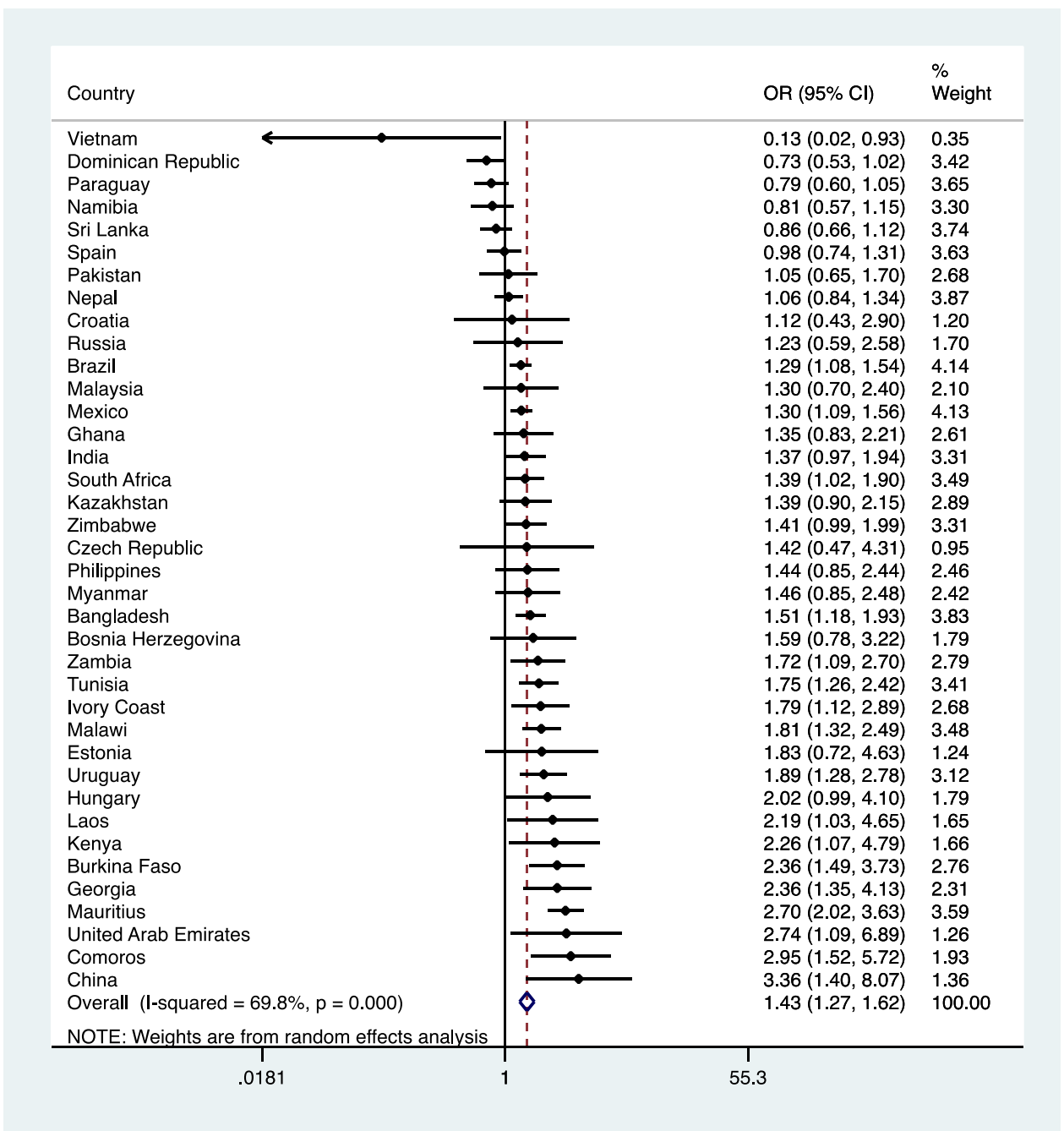

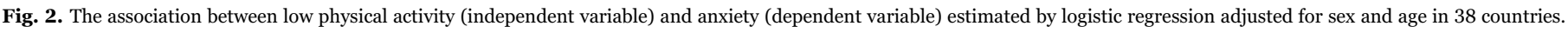
Abbreviation: OR odds ratio; CI confidence interval. Restricted to those aged 18-69 years. Overall pooled estimate was based on meta-analysis with random effects.

delivery. For example, effectiveness trials in different cultural settings could explore whether assisting people with anxiety in fulfilling three universal, psychological needs: (a) the need for autonomy (i.e., experiencing a sense of psychological freedom when engaging in exercise), (b) the need for competence (i.e., ability to attain desired outcomes following the exercise program), and (c) the need for relatedness (i.e., being socially connected when being physically active) will increase the likelihood that they adopt or maintain an active lifestyle (Vancampfort et al., 2015). Ministries of health and education play a critical role in governance and policy development. Physical activity needs to be mainstreamed in existing health policies and strategies at all levels of intervention including primary health care. Furthermore, governments should seek ways to provide appropriate environments for physical activity including, space, infrastructure and tools. Finally, governmental and non-governmental agencies will do well to increase public health awareness of the importance of physical activity in people with anxiety. For example, physical activity should be integrated into the existing Information, Education and Communication (IEC), especially on mental health and lifestyle diseases public health awareness programs of the World Health Organization (Clift, 2001). Targeted and regular messages should be developed in order to make such campaigns affordable. The benefits of engaging in physical activity in people with anxiety should be properly outlined; any fears and wrongly held beliefs within various cultural contexts dispelled and dealt with, precautions to take as regards to starting at a low intensity and ways to maintain an active lifestyle should be included in the awareness programs across communities and policy makers.

\subsection{Limitations}

The current findings should be interpreted in light of some limitations. First, the study is cross-sectional, therefore the directionality of the relationships we observed cannot be deduced readily. For example, it remains unclear whether lack of physical activity was caused by anxiety or vice versa. Second, although the response rate of the survey was high, our results may still be prone to Berkson's bias. For example, more anxious and/ or suspicious individuals may not have taken part in the WHS.. In addition, the fact that institutionalized individuals were excluded from the survey may also mean that individuals with higher levels of anxiety or low physical activities were not included in the analysis. Thus, our results may not be representative of severe cases of anxiety or low physical activity. Third, the anxiety variable used was based on a single question. However, this question has been used in previous WHS publications to define anxiety (Koyanagi and Stickley, 2015; Wong et al., 2013), and the use of extreme categories may have improved specificity. Moreover, there was variation in the prevalence of anxiety across different countries, which has previously been reported (Baxter et al., 2014). Fourth, our sample included a relatively low prevalence of insufficiently active adults of 
$17.3 \%$ when compared to previous global calculations of $31.1 \%$ (Hallal et al., 2012). However, this is explained by the use of the IPAQ measurement tool for our data, which is widely known to over-report PA participation and was adjusted accordingly in previous global estimates. Fifth, we only adjusted for depression as a co-morbid mental health condition and did not consider other comorbidities such as bipolar disorder. Future research may wish to investigate in detail the influence of additional mental health comorbidities on the relationship between anxiety and physical activity. Sixth, the sample was restricted to 18-69 and it remains unclear if the relationships we observed are similar in people outside of this range. Furthermore, the WHS was conducted over a decade ago and our results may not reflect the current situation in some countries. Finally, it is important to note that the cutpoints used for defining the physical activity prevalence into low, moderate and high categories corresponded to previous global recommendations that were based primarily on epidemiological evidence for its cardiorespiratory and metabolic health-related impact. We applied these cut-points because they most closely approximated existing evidence for the global prevalence of physical activity (Hallal et al., 2012), and as yet, there is limited dose-response research to formulate specific physical activity recommendations for mental health outcomes. Nonetheless, the strengths of the study include the multi-national scope, including most regions of the world. Moreover, the present study was performed with nationally representative samples of non-institutionalized persons.

In conclusion, the current study demonstrates high global levels of anxiety. Our global data also suggests that anxiety is associated with less physical activity. Physical activity needs to be mainstreamed in existing health policies and strategies at all levels of intervention including primary health care. To this end, policy makers and budget holders should invest in physical activity as part of a multidisciplinary treatment package while professionals should be able to improve their mental health skills. Finally, (inter)national agencies should increase public health awareness of the importance of physical activity across all people due to the established benefits but also include those with anxiety who are at particular risk of inactivity, which may exacerbate ill health further. Providing the financial resources to deliver these interventions and awareness campaigns may prove cost-effective through reducing the sizeable economic health burden associated with physical inactivity.

\section{Conflict of interest}

BS, AK, SR, DV FS, JR have no conflicts of interest to declare.

\section{Acknowledgments}

BS receives funding from National Institute for Health Research Collaboration for Leadership in Applied Health Research \& Care Funding scheme. The views expressed in this publication are those of the author(s) and not necessarily those of the NHS, the National Institute for Health Research or the Department of Health.

AK's work was supported by Miguel Servet Contract financed by the CP13/ 00,150 and PI15/00862 projects, integrated into the National R $+\mathrm{D}+\mathrm{I}$ and funded by ISCIII - General Branch Evaluation and Promotion of Health Research - and European Regional Development Fund (ERDF-FEDER).

SR is funded by a Society for Mental Health Research Early Career Fellowship (Australia).

DV is funded by Research Foundation - Flanders (FWO Vlaanderen).

JR is funded by an NHMRC Early Career Fellowship (Australia).

\section{References}

anxiety disorder. Curr. Top. Behav Neurosci. 2, 453-467.

Baldwin, D.S., Waldman, S., Allgulander, C., 2011. Evidence-based pharmacological treatment of generalized anxiety disorder. Int. J. Neuropsychopharmacol. 14 (5), $697-710$.

Batelaan, N.M., Seldenrijk, A., Bot, M., van Balkom, A.J., Penninx, B.W., 2016. Anxiety and new onset of cardiovascular disease: critical review and meta-analysis. Br. J. Psychiatry 208 (3), 223-231.

Baxter, A.J., Scott, K.M., Vos, T., Whiteford, H.A., 2013. Global prevalence of anxiety disorders: a systematic review and meta-regression. Psychol. Med. 43 (5), 897-910.

Baxter, A.J., Vos, T., Scott, K.M., Ferrari, A.J., Whiteford, H.A., 2014. HThe global burden of anxiety disorders in 2010. Psychol. Med. 44 (11), 2363-2374.

Caldirola, D., Schruers, K.R., Nardi, A.E., De Berardis, D., Fornaro, M., Perna, G., 2016 Is there cardiac risk in panic disorder? An updated systematic review. J. Affect Disord. 194, 38-49.

Chisholm, D., Sweeny, K., Sheehan, P., et al., 2016. Scaling-up treatment of depression and anxiety: a global return on investment analysis. Lancet Psychiatry 3 (5), 415-424.

Clift, E., 2001. Information education and communication: lessons from the past; perspectives for the future.

Conn, V.S., 2010. Anxiety outcomes after physical activity interventions: meta-analysis findings. Nurs. Res. 59 (3), 224-231.

Craig, C., Marshall, A., Sjostrom, M., et al., 2003. International physical activity questionnaire: 12-country reliability and validity. Med. Sci. Sports Exerc. 35 (8), 1381-1395.

Craig, C.L., Marshall, A.L., Sjöström, M., et al., 2003. International physical activity questionnaire: 12-country reliability and validity including commentary by Bassett DR Jr. Med. Sci. Sports Exerc. 35 (8), 1381-1396.

Da Silva, M.A., Singh-Manoux, A., Brunner, E.J., et al., 2012. Bidirectional association between physical activity and symptoms of anxiety and depression: the Whitehall II study. Eur. J. Epidemiol. 27 (7), 537-546.

De Herdt, A., Knapen, J., Vancampfort, D., De Hert, M., Brunner, E., Probst, M., 2013 Social anxiety in physical activity participation in patients with mental illness: a cross-sectional multicenter study. Depress Anxiety 30 (8), 757-762.

Dworak, M., Schierl, T., Bruns, T., Struder, H.K., 2007. Impact of singular excessive computer game and television exposure on sleep patterns and memory performance of school-aged children. Pediatrics 120 (5), 978-985.

Edwards, M.K., Loprinzi, P.D., 2016. Experimentally increasing sedentary behavior results in increased anxiety in an active young adult population. J. Affect Disord. 204, 166-173.

Endrighi, R., Steptoe, A., Hamer, M., 2015. The effect of experimentally induced sedentariness on mood and psychobiological responses to mental stress. Br. J. Psychiatry.: J. Ment. Sci..

Hallal, P.C., Andersen, L.B., Bull, F.C., Guthold, R., Haskell, W., Ekelund, U., 2012 Global physical activity levels: surveillance progress, pitfalls, and prospects. Lancet 380 (9838), 247-257.

Haller, H., Cramer, H., Lauche, R., Gass, F., Dobos, G.J., 2014. The prevalence and burden of subthreshold generalized anxiety disorder: a systematic review. BMC Psychiatry 14, 128.

Helgadóttir, B., Forsell, Y., Ekblom, Ö., 2015. Physical activity patterns of people affected by depressive and anxiety disorders as measured by accelerometers: a cross-sectional study. PloS One 10 (1), e0115894.

Herring, M.P., O'Connor, P.J., Dishman, R.K., 2010. The effect of exercise training on anxiety symptoms among patients: a systematic review. Arch. Int. Med. 170 (4), $321-331$.

Hoare, E., Skouteris, H., Fuller-Tyszkiewicz, M., Millar, L., Allender, S., 2014. Associations between obesogenic risk factors and depression among adolescents: a systematic review. Obes. Rev. 15 (1), 40-51.

Hoffman, D.L., Dukes, E.M., Wittchen, H.U., 2008. Human and economic burden of generalized anxiety disorder. Depress Anxiety 25 (1), 72-90.

Hofmann, S.G., Smits, J.A., 2008. Cognitive-behavioral therapy for adult anxiety disorders: a meta-analysis of randomized placebo-controlled trials. J. Clin. Psychiatry 69 (4), 621-632.

Jayakody, K., Gunadasa, S., Hosker, C., 2013. Exercise for anxiety disorders: systematic review. Br. J. Sports Med 48 (3), 187-196.

Koyanagi, A., Stickley, A., 2015. The association between sleep problems and psychotic symptoms in the general population: aa global perspective. Sleep 38 (12), $1875-1885$.

Lewis, C., Pearce, J., Bisson, J.I., 2012. Efficacy, cost-effectiveness and acceptability of self-help interventions for anxiety disorders: systematic review. Br. J. Psychiatry 200 (1), 15-21.

Ma, W.F., Yen, W.J., Chen, W.C., Chang, H.J., Huang, X.Y., Lane, H.Y., 2009. Roles of state and trait anxiety in physical activity participation for adults with anxiety disorders. J. Formos. Med Assoc. 108 (6), 481-492.

Mendlowicz, M.V., Stein, M.B., 2000. Quality of life in individuals with anxiety disorders. Am. J. Psychiatry 157 (5), 669-682.

Nuevo, R., Chatterji, S., Verdes, E., Naidoo, N., Arango, C., Ayuso-Mateos, J.L., 2012. The continuum of psychotic symptoms in the general population: a cross-national study. Schizophr. Bull. 38 (3), 475-485.

Olatunji, B.O., Cisler, J.M., Tolin, D.F., 2007. Quality of life in the anxiety disorders: a meta-analytic review. Clin. Psychol. Rev. 27 (5), 572-581.

Park, S., Cho, M.J., Cho, S.-J., et al., 2011. Relationship between physical activity and mental health in a nationwide sample of Korean adults. Psychosomatics 52 (1) 65-73.

Pedersen, B., Saltin, B., 2015. Exercise as medicine-evidence for prescribing Exercise as therapy in 26 different chronic diseases. Scand. J. Med. Sci. Sports 25 (S3), 1-72.

Penedo, F.J., Dahn, J.R., 2005. Exercise and well-being: a review of mental and physical 
health benefits associated with physical activity, Curr. Opin. Psychiatry 18 (2), 189-193.

Prina, A.M., Ferri, C.P., Guerra, M., Brayne, C., Prince, M., 2011. Co-occurrence of anxiety and depression amongst older adults in low- and middle-income countries: findings from the 10/66 study. Psychol. Med 41 (10), 2047-2056.

Rebar, A.L., Duncan, M.J., Short, C., Vandelanotte, C., 2014. Differences in healthrelated quality of life between three clusters of physical activity, sitting time, depression, anxiety, and stress. BMC Public Health 14, 1088.

Roest, A.M., Zuidersma, M., de Jonge, P., 2012. Myocardial infarction and generalised anxiety disorder: 10-year follow-up. Br. J. Psychiatry 200 (4), 324-329.

Rubin, K.H., 2001. In: Vasey, M.W., Dadds, M.R. (Eds.), KB B. The developmental psychopathology of anxiety. Oxford University Press.

da Silva, C.T.B., Schuch, F., Costa, M., Hirakata, V., Manfro, G.G., 2014. Somatic, but not cognitive, symptoms of anxiety predict lower levels of physical activity in panic disorder patients. J. Affect. Disord. 164, 63-68.

Stubbs, B., Vancampfort, D., Rosenbaum, S., et al. An examination of the anxiolytic effects of exercise for people with anxiety and stress-related disorders: A metaanalysis Psychiatry Res. Submitted for publication.

Stubbs, B., Aluko, Y., Myint, P.K., Smith, T.O., 2016a. Prevalence of depressive symptoms and anxiety in osteoarthritis: a systematic review and meta-analysis. Age Ageing 45 (2), 228-235.

Stubbs, B., Vancampfort, D., Rosenbaum, S., et al., 2016b. Dropout from exercise randomized controlled trials among people with depression: a meta-analysis and meta regression. J. Affect. Disord. 190, 457-466.

Teychenne, M., York, R., 2013. Physical activity, sedentary behavior, and postnatal depressive symptoms: a review. Am. J. Prev. Med 45 (2), 217-227.

Teychenne, M., Ball, K., Salmo, J., 2010. Sedentary behavior and depression Among adults: a review. Int. J. Behav. Med. 17 (4), 246-254.

Teychenne, M., Costigan, S.A., Parker, K., 2015. The association between sedentary behaviour and risk of anxiety: a systematic review. BMC Public Health 15, 513.

Vancampfort, D., Stubbs, B., Venigalla, S.K., Probst, M., 2015. Adopting and maintaining physical activity behaviours in people with severe mental illness: the importance of autonomous motivation. Prev. Med.

Vancampfort, D., Rosenbaum, S., Schuch, F., et al., 2016a. Cardiorespiratory fitness in severe mental illness: aa systematic review and meta-analysis. Sports Med.

Vancampfort, D., Rosenbaum, S., Schuch, F.B., Ward, P.B., Probst, M., Stubbs, B., 2016b. Prevalence and predictors of treatment dropout from physical activity interventions in schizophrenia: a meta-analysis. Gen. Hosp. Psychiatry 39, 15-23.

de Vries, Y.A., de Jonge, P., van den Heuvel, E., Turner, E.H., Roest, A.M., 2016. Influence of baseline severity on antidepressant efficacy for anxiety disorders: metaanalysis and meta-regression. Br. J. Psychiatry 115, 173450.

Wang, X., Perry, A.C., 2006. Metabolic and physiologic responses to video game play in 7- to 10-year-old boys. Arch. Pediatr. Adolesc. Med. 160 (4), 411-415.

Wong, K.O., Hunter Rowe, B., Douwes, J., Senthilselvan, A., 2013. Asthma and wheezing are associated with depression and anxiety in adults: an analysis from 54 countries. Pulm. Med. 2013, 929028. 